OPEN ACCESS

Edited by:

Chunfu Zheng,

University of Calgary, Canada

Reviewed by:

Bin Li,

Jiangsu Academy of Agricultural

Sciences (JAAS), China

Gaopeng Hou,

Washington University School

of Medicine in St. Louis, United States

*Correspondence:

Baicheng Huang

hbch228@163.com

Yani Sun

sunyani@nwsuaf.edu.cn

Specialty section:

This article was submitted to

Virology,

a section of the journal

Frontiers in Microbiology

Received: 20 December 2021

Accepted: 03 January 2022

Published: 21 January 2022

Citation:

Li N, Zhang Y, Yao L, Shi Y,

Zhao Q, Huang B and Sun Y (2022) A

Recombinant Porcine Reproductive and Respiratory Syndrome Virus

Stably Expressing DsRed Protein

Based on Bacterial Artificial

Chromosome System

Front. Microbiol. 13:839845.

doi: 10.3389/fmicb.2022.839845

\section{A Recombinant Porcine} Reproductive and Respiratory Syndrome Virus Stably Expressing DsRed Protein Based on Bacterial Artificial Chromosome System

\author{
Na Li', Yiyi Zhang', Lunguang Yao', Yunpeng Shi², Qin Zhao ${ }^{3}$, Baicheng Huang ${ }^{4 *}$ and \\ Yani Sun ${ }^{3 *}$ \\ ' Key Laboratory of Ecological Security, Collaborative Innovation Centre of Water Security for Water Source Region \\ of Mid-line of South-to-North Diversion Project of Henan Province, Henan Provincial Engineering and Technology Center \\ of Health Products for Livestock and Poultry, School of Life Sciences and Agricultural Engineering, Nanyang Normal \\ University, Nanyang, China, ${ }^{2}$ Shijiazhuang Customs (Huanghua Port), Cangzhou, China, ${ }^{3}$ College of Veterinary Medicine, \\ Northwest A\&F University, Yangling, China, ${ }^{4}$ National Research Center for Veterinary Medicine, Luoyang, China
}

Recombinant viruses possessing reporter proteins as tools are widely applied in investigating viral biology because of the convenience for observation. Previously, we generated a recombinant pathogenic porcine reproductive and respiratory syndrome virus (PRRSV) with enhanced green fluorescent protein (EGFP) reporter for monitoring virus spread and screening of neutralizing antibodies. PRRSV with different kinds of reporters can support more application scenarios. Here, we described a new genetically stable infectious clones of a highly pathogenic PRRSV (HP-PRRSV) harboring the DsRed (a red fluorescent protein isolated from the coral Discosoma) gene. In the recombinant infectious clone, the transcription regulatory sequence 2 (TRS2) of PRRSV was inserted between the open reading frame 7 (ORF7) and $3^{\prime}$ UTR to drive the transcription of DsRed gene, which makes it a separate transcription unit in the viral genome. Using the bacterial artificial chromosome (BAC) system and cytomegalovirus (CMV) promoter, the recombinant HP-PRRSV with the DsRed insertion was successfully rescued and showed similar growth and replication patterns compared with the wildtype virus in the MARC-145 cells. In addition, the DsRed protein was stably expressed in the recombinant virus for at least 10 passages with consistent fluorescence intensity and density. Using the recombinant HP-PRRSV with DsRed protein, the virus tracking in MARC-145 was observed by live-cell imaging. Meanwhile, quantification of the DsRed fluorescence positive cells by flow cytometry provides an alternative to standard methods for testing the level of PRRSV infection. This recombinant PRRSV with DsRed fluorescence protein expression could be a useful tool for fundamental research on the viral biology and shows the new design for stable expression of foreign genes in PRRSV.

Keywords: HP-PRRSV, DsRed, bacterial artificial chromosome, transcription regulatory sequence, reporter 


\section{INTRODUCTION}

Porcine reproductive and respiratory syndrome (PRRS) is a widespread disease affecting domestic pigs, first reported in North America and Europe in the late 1980s (Wensvoort et al., 1991; Albina, 1997). The symptoms of PRRS include reproductive failure, pneumonia, and increased susceptibility to secondary bacterial infection. The causative agent of PRRS is the PRRS virus (PRRSV), which is in the Arteriviridae family of the order Nidovirales. PRRSV is an enveloped, singlestranded positive-sense RNA virus of approximately $15 \mathrm{~kb}$ in length that contains 9 open reading frames (ORFs) (Fang and Snijder, 2010). Two distinct viral genotypes of PRRSV are North American type and European type, only shared the genome identity by approximately $60 \%$. In the early 2000 s, the outbreak of highly pathogenic PRRS (HP-PRRS) inflicted serious economic losses, which characterized by high-grade fever with increased mortality in pigs of all ages (Tian et al., 2007). The modified attenuated and killed vaccines have been wildly used in the field (Charerntantanakul, 2012). However, there is no remarkably effective vaccine for controlling PRRSV infection in pigs.

In the past decade, reverse genetics technology was applied to many RNA viruses to generate recombinant mutant viruses for virology and biology studies (Racaniello and Baltimore, 1981; Bernstein et al., 1986; Gonzalez et al., 2002; Almazan et al., 2006; St-Jean et al., 2006; Park et al., 2012; Amarilla et al., 2021; Nouda et al., 2021). Many infectious clones of different PRRSV strains have been constructed, including North American and European type strains (Nielsen et al., 2003; Truong et al., 2004; Fang et al., 2006; Zhang et al., 2011; Sang et al., 2012; Wang et al., 2013). In various rescue systems, the bacterial artificial chromosomes (BAC) have the advantages of large antigen-capacity, high fidelity in replication, and the easier procedures of manipulation, which was applied both in DNA (Messerle et al., 1997; Borst et al., 1999; Suter et al., 1999; Kanda et al., 2004) and RNA virus (Almazan et al., 2000; Almazan et al., 2006; Wang et al., 2013). The reverse genetics technology with fluorescence protein insertion is a common strategy for recombinant virus labeling, which gives an intuitive convenience for virus observation by fluorescence microscopy or flow cytometer. The application of fluorescence proteins in virus labeling mainly focuses on the enhanced green fluorescent protein (EGFP). Previously, we generated a recombinant PRRSV with EGFP expression, and the transcription regulatory sequence for ORF6 (TRS6) was inserted between the ORF7 and $3^{\prime}$ UTR to drive the transcription of the EGFP (Wang et al., 2013). The results showed that the recombinant PRRSV with EGFP was a useful tool for neutralizing antibodies screening. However, to study the real-time interaction of various proteins, it is necessary to achieve multi-fluorescence labeling for live-cell imaging (Wolff et al., 2006; Strickfaden et al., 2010; Behrens et al., 2017).

In this study, to produce a differentiated recombinant PRRSV for fluorescence capture, we developed an HP-PRRS virus (HPPRRSV) infectious clone with a red fluorescence protein of DsRed (a red fluorescent protein isolated from the coral Discosoma) based on BAC system. The DsRed gene was inserted between the
ORF7 gene and the $3^{\prime} \mathrm{UTR}$ region of the PRRSV genome and transcripted by TRS2 (the sequence length of TRS2 is shorter than that of TRS6). The expression levels and stability of the DsRed in the rescued recombinant PRRSV were investigated, in addition, the virus tracking in MARC-145 and the quantification of the DsRed fluorescence positive cells by flow cytometry were also studied.

\section{MATERIALS AND METHODS}

\section{Plasmid and Cell Lines}

The PRRSV infectious clone plasmid pBAC-SD $16^{F L}$-AM containing the whole viral genome derived from the HP-PRRSV strain (SD16 strain, GenBank: JX087437) was constructed based on the results on previous descriptions (Wang et al., 2013). MARC-145 cell line, derived from the embryonic African green monkey kidney cell line (MA-104), was cultured in the Dulbecco's modified Eagle's medium (DMEM) supplemented with 10\% fetal bovine serum (FBS) (HyClone, South Logan, UT, United States) at $37^{\circ} \mathrm{C}$ in a $5 \% \mathrm{CO}_{2}$ incubator.

\section{Plasmid Construction With DsRed Gene Insertion}

The plasmid with DsRed gene insertion was constructed as previously described (Wang et al., 2013). Briefly, the DsRed gene was amplified from pDsRed-Express-N1 Vector (Clontech, CA, United States) with primers $5^{\prime}$-GCGATCGC TTGAACCAACTTTAGGCCTGAATTGAAATGGCCTCCTCCG AGGC-3' (the AsisI site is underlined and TRS2 sequence is in italics), and 5'-CAGCCCACGACGCGTCGCTACAGGAA CAGGTGGTG-3' (the MluI site is underlined) by polymerase chain reaction (PCR). The amplified product was inserted into the pEasy $^{\mathrm{TM}}$-blunt simple cloning vector to generate plasmids pEasy-TRS2-DsRed. After sequencing, the positive plasmids containing TRS2-DsRed genes and pBAC-SD16 ${ }^{F L}$-AM were digested with AsiSI and MluI, and then the digested products were ligated to generate plasmid pBAC-SD16 $6^{F L}$-TRS2-DsRed. The design of the recombinant PRRSV infection clone is shown in Figure 1A. The recombinant plasmid was identified by sequencing. Finally, the positive plasmid was extracted with the QIAfilter Plasmid Midi Kit (QIAGEN, Hilden, Germany) and transfected into MARC-145 cells.

\section{Transfection and Rescue of Recombinant Viruses}

The recombinant PRRSV carrying DsRed was rescued according to the method in previous report (Wang et al., 2013). Briefly, MARC-145 cells in a 6-well plate ( $80 \%$ confluent) were transfected with the plasmids pBAC-SD16 ${ }^{F L}$-TRS2-DsRed using Attractene Transfection Reagent (QIAGEN, Hilden, Germany) following the manufacturers' instructions. After 2 days of incubation at $37^{\circ} \mathrm{C}$ and $5 \% \mathrm{CO}_{2}$, the cytopathic effect (CPE) in cells were checked daily, and the fluorescence was captured when the $\mathrm{CPE}$ was found. In addition, the culture supernatants from the transfected cells were used to infect MARC-145 cells to 
A

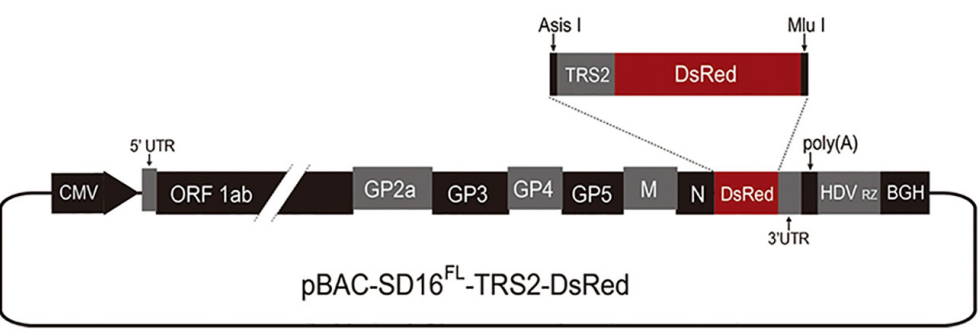

B

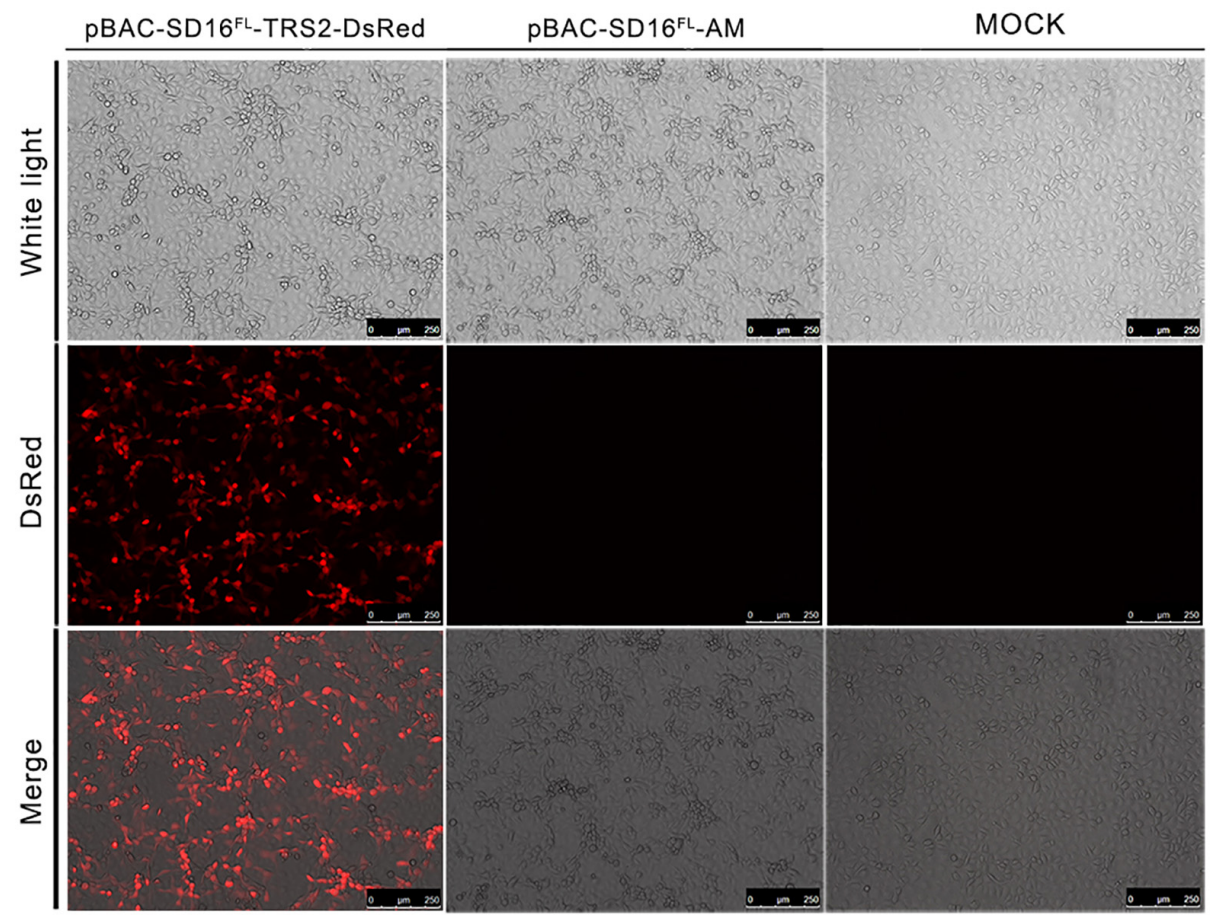

FIGURE 1 | Plasmid construction of pBAC-SD16 FL -TRS2-DsRed and DsRed expression in MARC-145 cells after transfection. (A) The DsRed gene was flanked by the transcription regulatory sequence 2 (TRS2) at the $5^{\prime}$ end was inserted into plasmid pBAC-SD16 ${ }^{F L}$-AM to produce plasmid pBAC-SD16 ${ }^{F L}$-TRS2- DsRed using sites of AsiSI and Mlul; (B) MARC-145 cells transfected with pBAC-SD16 FL-TRS2-DsRed or pBAC-SD16 ${ }^{F L}$-AM in a 6-well plate, after $48 \mathrm{~h}$ post-transfection, the fluorescence pictures in the live cells were captured by fluorescence microscopy.

propagate the rescued virus. The rescued viruses were confirmed by sequencing. The rescued virus was named rSD16/TRS2DsRed.

\section{Immunofluorescence Assays}

MARC-145 cells were cultured on glass coverslips in a 24-well plate and were infected with SD16 or rSD16/TRS2-DsRed (3rd passage) at a multiplicity of infection (MOI) of 0.01 for $48 \mathrm{~h}$. Cells were fixed with $4 \%$ formaldehyde for $30 \mathrm{~min}$ at room temperature (RT) and permeabilized with $0.5 \%$ Triton X-100 in phosphate-buffered saline (PBS) for $10 \mathrm{~min}$. Then, the cells were incubated with monoclonal antibody $(\mathrm{mAb})$ against PRRSV nucleocapsid $(\mathrm{N})$ protein $(6 \mathrm{D} 10)$ for $1 \mathrm{~h}$ at room temperature as previously described (Li et al., 2016). After that, the cells were still incubated with Alexa Fluor ${ }^{\circledR} 488$ AffiniPure Goat Anti-Mouse IgG (Jackson, West Grove, PA, United States) for $1 \mathrm{~h}$ at RT. Finally, the cells were stained with 4-6-diamidino-2-phenylindole
(DAPI) for $5 \mathrm{~min}$ at RT. Images were taken by Leica microscope. Mock-infected MARC-145 cells were used as controls.

\section{Western Blotting}

MARC-145 cells were infected with SD16 or rSD16/TRS2-DsRed (3rd passage) at an MOI of 0.01 . When $60 \%$ of the cells showed $\mathrm{CPE}$, the infected cells were collected and lysed. The cellular proteins were separated by SDS-PAGE and transferred on a polyvinylidene difluoride (PVDF) membrane. The membrane was incubated with anti- $\alpha$-Tubulin antibody (Sigma-Aldrich, MO, United States), mouse anti-DsRed mAb (Santa Cruz Biotechnology, United States) and 6D10 mAb overnight at $4^{\circ} \mathrm{C}$. And then, it was incubated with peroxidase-conjugated goat anti-mouse IgG (Jackson, PA, United States) for $1 \mathrm{~h}$ at RT. Immuno-stained proteins were visualized using ECL Western Blotting Substrate (Pierce, CA, United States). Cellular proteins from mock-infected MARC-145 cells were used as controls. 


\section{Imaging of Porcine Reproductive and Respiratory Syndrome Virus With DsRed Expression}

MARC-145 cells were grown on a $35 \mathrm{~mm}$ cell culture dish to a density of $50-70 \%$ confluence and then infected with rSD16/TRS2-DsRed (MOI: 0.1) of different passages. After $1 \mathrm{~h}$ post-infection (hpi), the medium was removed and replaced with $2 \mathrm{~mL}$ of pre-warmed DMEM containing 3\% FBS. The dish was placed at $37^{\circ} \mathrm{C}$ with $5 \% \quad \mathrm{CO}_{2}$. The fluorescence of different passages of rSD16/TRS2-DsRed was captured by Leica microscope at $36 \mathrm{hpi}$. In live-cell imaging, MARC-145 cells were infected with rSD16/TRS2/DsRed at an MOI of 0.01 in a $35 \mathrm{~mm}$ cell culture dish. At $1 \mathrm{hpi}$, the medium was removed and replaced with $2 \mathrm{ml}$ of pre-warmed DMEM containing 3\% FBS. The dish was placed in the $37^{\circ} \mathrm{C}$ observation chamber containing $5 \% \mathrm{CO}_{2}$ (Leica CTR-Controller 3700, Wetzlar, Germany). DsRed signals and phase-contrast images were captured with the time interval of $1 \mathrm{~min}$ for $3 \mathrm{~h}$ by a live-cell station (Leica AF6000, Wetzlar, Germany). Subsequently, images were processed into a movie of 10 frames s-1 using QuickTime Pro.

\section{Determination of Growth Curves for Recombinant Porcine Reproductive and Respiratory Syndrome Virus}

Recombinant virus rSD16/TRS2-DsRed was propagated in MARC-145 cells with an MOI of 1.0. The growth curves of rSD16/TRS2-DsRed and parental virus SD16 were compared. Briefly, $1 \mathrm{~h}$ after virus adsorption, MARC-145 cells were washed three times with PBS and incubated in DMEM with 3\% FBS at $37^{\circ} \mathrm{C}, 5 \% \mathrm{CO}_{2}$. The supernatants were collected at various time points $(12,24,36,48,60$, and $72 \mathrm{hpi})$, and viral titers were calculated based on the $\mathrm{TCID}_{50}$ data (Calculate by ReedMuench method).

\section{Polymerase Chain Reaction Analysis of Viral RNA Isolation and Reverse Transcription}

To determine the mRNA production of the DsRed gene in different passages, the viral RNA of rSD16/TRS2-DsRed (passages 1, 3, 5, 7, and 9) was extracted from supernatants of culture medium with the RNAiso Plus (Takara, Dalian, China) and then reverse-transcribed into cDNAs with M-MLV reverse transcriptase (Takara, Dalian, China). The target PCR fragments were amplified using the primer pairs of $5^{\prime}$ ATACTGTGCGCCTGATCCGC-3' (located in the $3^{\prime}$ end of $\mathrm{N}$ protein) and $5^{\prime}$ - TCGCCAATTAAACTTTACCCCCACA- $3^{\prime}$ (located in the $5^{\prime}$ end of $3^{\prime} \mathrm{UTR}$ region).

\section{RESULTS}

\section{Rescue of rSD16/Transcription Regulatory Sequence 2-DsRed}

A vector pBAC-SD $16^{F L}-\mathrm{AM}$ with $A$ siSI and $M l u I$ sites between the ORF7 gene and the $3^{\prime}$ UTR region of the viral genome was used to construct PRRSV infectious clone with DsRed. The
DsRed gene was flanked by the unique AsiSI and MluI sites for its insertion into the plasmid pBAC-SD $16^{F L}-\mathrm{AM}$, and its expression was driven by the transcription-regulating sequence of gene GP2a (TRS2) (Figure 1A). An apparent DsRed fluorescence was observed in the MARC-145 cells after transfection with pBACSD16 ${ }^{F L}$-TRS2-DsRed (Figure 1B), which means the successful expression of indictor protein.

\section{Replication Properties of Recombinant Viruses}

The expression of DsRed and PRRSV N protein in MARC-145 cells infected with rSD16/TRS2-DsRed were identified by western blot using anti-DsRed and anti-PRRSV $\mathrm{N}$ protein $\mathrm{mAbs}$ as primary antibodies. As shown in Figure 2A, DsRed and PRRSV $\mathrm{N}$ protein were detected in rSD16/TRS2-DsRed infected cells, and DsRed was not detected in SD16-infected or normal MARC145 cells. PRRSV N protein was also detected in virus-infected cells by immunofluorescence assays but not in the normal cells (Figure 2C). The replication ability of the recombinant virus with DsRed insertion was also compared by growth curves of SD16 and rSD16/TRS2-DsRed in MARC-145 cells. The supernatant of the culture medium of rSD16/TRS2-DsRed and the parental virus were collected at $12,24,36,48$, and $60 \mathrm{hpi}$, respectively. The viral titers were examined at the indicated time points. As shown in Figure 2B, the growth kinetics of recombinant PRRSV rSD16/TRS2-DsRed was not significantly different from the parental virus, and the titers peaked at $48 \mathrm{hpi}$ for both viruses.

\section{Stability of DsRed Expression in Recombinant Viruses}

The recombinant viruses were passaged in MARC-145 cells for at least 10 passages to investigate the stability of DsRed expression. As visualized by fluorescence microscopy, positive cells exhibited a sustained red fluorescence at different passages (Figure 3A). The viral titers of the recombinant virus in different passages were also detected by the TCID 50 method. As shown in Figure 3B, no significant changes of the viral titers were found in different passages. Furthermore, the presence of the DsRed gene in the viral genome was identified by RT-PCR. As shown in Figure 3C, all fragments were consistent with the expected length of about $750 \mathrm{bp}$. In addition, the PCR products were also sequenced, and the results showed no mutations in the DsRed gene (data not shown).

\section{Intercellular Spread of rSD16/Transcription Regulatory Sequence 2/DsRed}

Live-cell imaging was performed in recombinant virus-infected MARC-145 cells to determine whether the rSD16/TRS2/DsRed could be used as a reporter virus. As shown in Supplementary Movie 1, the DsRed fluorescent in the recombinant virusinfected cells could be observed at approximately $24 \mathrm{hpi}$, and the fluorescence is distributed evenly in the cells. We also found that the DsRed fluorescence could be transported along intercellular connecting nanotube to neighboring cells. These results suggested that the recombinant PRRSV rSD16/TRS2/DsRed 
A

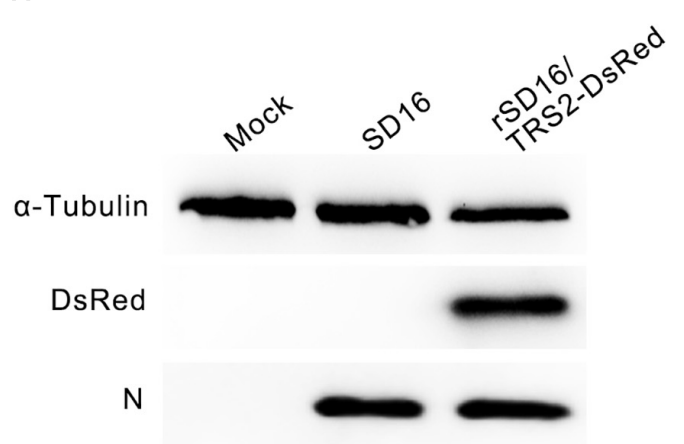

B

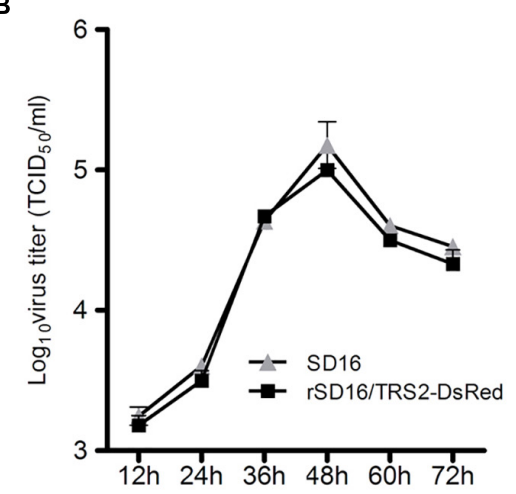

C

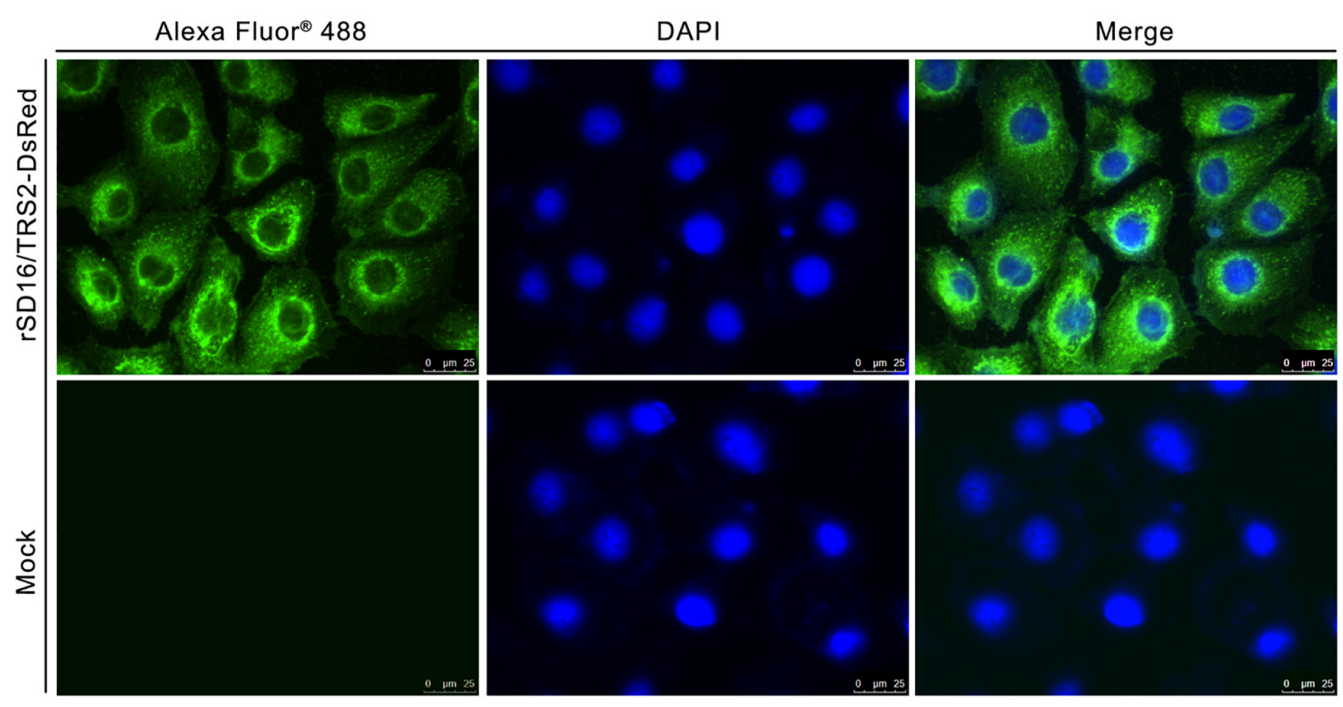

FIGURE 2 | DsRed protein expression and growth curve of rSD16/TRS2-DsRed. (A) After being infected with SD16 or rSD16/TRS2-DsRed (MOI of 0.1) for 36 h, the MARC-145 cells were lysed for the western blot analysis of PRRSV protein and DsRed expression, the MARC-145 cells infected with SD16 and normal MARC-145 cells were taken as the control; (B) MARC-145 cells were infected with SD16 or rSD16/TRS2-DsRed at the MOl of 1.0, and the progeny virus produced in the culture medium was measured at a series of times from 12 to $72 \mathrm{hpi}$ by the method of TCID 50 detection, and all the data were expressed as the mean \pm standard deviation (SD); (C) after infected with rSD16/TRS2-DsRed for $48 \mathrm{~h}$, the MARC-145 cells were fixed for the immunofluorescence analysis using the monoclonal antibody of PRRSV N protein, the normal MARC- 145 cells were the control.

can be used as a reporter virus to analyze the biological characterizations of PRRSV.

\section{DsRed as an Indicator of Recombinant Virus Infection}

To validate the correlation between the DsRed and the viral infection, the DsRed fluorescence intensity from the infected virus was measured by flow cytometry. As shown in Figure 4A, a gradient manner of red fluorescence positive cells ratio in MARC145 cells infected with rSD16/TRS2/DsRed in different hpi. The ratio of positive cells ranged from $5.49 \%$ at hpi of 24 (MOI: 0.1 ) to $35.72 \%$ at hpi of 48 (MOI: 1.0 ), indicating a high ratio of DsRed positive cells when the culture time was prolonged. Western blot analysis of PRRSV N protein and TCID $_{50}$ detection of culture supernatant were performed to verify whether the ratio of fluorescence positive cells is related to the virus infection. As shown in Figures $4 \mathbf{B}, \mathbf{C}$, the gradient manner is similar to the flow cytometry results of different hpi.

\section{DISCUSSION}

One of the most important achievements in RNA virus research is the development of reverse genetics systems, which makes the possibility of the virus genetic manipulation (Ishibashi et al., 2017). Reverse genetics technology has been extensively utilized to explore various aspects of virus infection, including infection mechanisms, virulence, pathogenesis, immune responses, transmission characteristics, vaccine development, and antiviral screening tests (Hoenen et al., 2011). There are two strategies to construct a reverse genetics system for PRRSV depending on whether viral RNA or cDNA is used for transfection (Chaudhari and $\mathrm{Vu}, 2020$ ). It has been demonstrated that the 
A

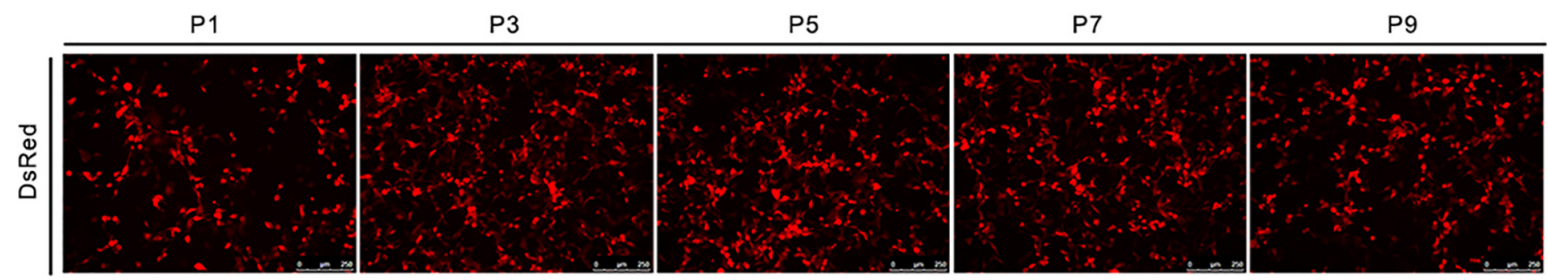

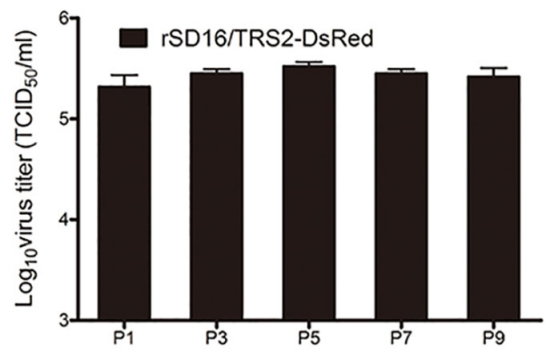

C

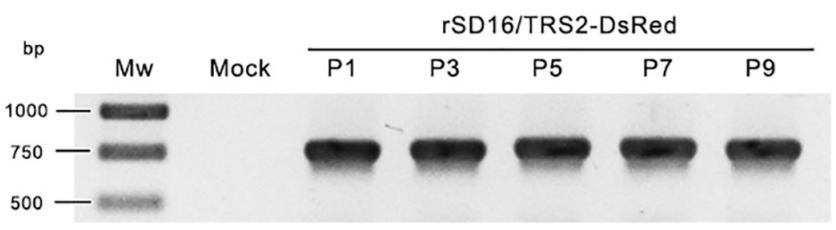

FIGURE 3 | Stable expression of DsRed in different passages of rSD16/TRS2-DsRed. (A) In different passages, fluorescence pictures of the recombinant virus were captured at 48 hpi by fluorescence microscopy; (B) titers of the recombinant virus were detected at different passages by TCID 50 method, all the tests were repeated three times; (C) mRNA production of DsRed gene at different passages of the recombinant virus were detected by reverse transcription PCR analysis.

DNA-based transfection system produces 10-100-fold higher viral titers than the RNA-based transfection system (Huang et al., 2009). The BAC system is based on the Escherichia coli (E. coli) F factor, in which replication is strictly controlled in E. coli. In BAC system, structural stability of inserted DNA in the host on the higher degree at the lower number of copies per cell (one or two per cell), beside the capability of maintaining large fragments (Shizuya et al., 1992), which applied in the rescue of many kinds of virus, comprising virus belongs to Coronaviridae (Almazan et al., 2000, 2006; Wang et al., 2013) and Herpesviridae (Messerle et al., 1997; Borst et al., 1999; Suter et al., 1999; Kanda et al., 2004; Li et al., 2011). Based on this system, we revealed that the $\sim 15.4 \mathrm{~kb}$ fragment of the PRRSV viral genome was inserted in the BAC plasmid for transfection more conveniently without steps like in vitro transcription.

Genetically recombinant viruses which express green and red fluorescent proteins are universally used as tools for studying replication and spread of diverse RNA viruses in living cells or animals (Kanai et al., 2019). Although PRRSV with EGFP has been generated (Wang et al., 2013), this is the first report of a recombinant HP-PRRSV expressing red fluorescence. DsRed, derived from the coral Discosoma, has an orange-red fluorescence and can form a stable tetramer, which is detectable by laser-based confocal microscopes and flow cytometers with an excitation at $568 \mathrm{~nm}$ (Hawley et al., 2001). It was possible to simultaneously measure the efficiency and fluorescence intensity of the transfected live cells. Compared with EGFP, DsRed has shown its tremendous advantages, including bright red fluorescence and high resistance against photo-bleaching (Garcia-Parajo et al., 2001). Recombinant viruses expressing DsRed proteins represent a better option to combine with genetically modified GFP-expressing cell lines or animals. Their reduced auto-fluorescence background captures the dynamics of viral infection and replication (Chiem et al., 2021). DsRed expression was used for the high-throughput hepatotoxicity test in drug screening and biomonitoring environmental toxicants in zebrafish (Zhang et al., 2014), the investigation of the host and tumor cell compartments (Jacobsen et al., 2013), and identification of MHC I-restricted epitope in mouse (Davey et al., 2013). In the cells, which were used in highthroughput scale detection of allergic sensitization (Wang et al., 2014), in vivo imaging of metastatic disease (Yu et al., 2012), genotoxicity, and oxidative stress assessment (Hendriks et al., 2011). As showed in this study, the DsRed fluorescence in the recombinant HP-PRRSV was observed in the MARC-145 cells with CPE (Figure 1).

Meanwhile, the growth kinetics of recombinant PRRSV rSD16/TRS2-DsRed showed no significant difference compared with that of the parental virus (Figure 2). The DsRed fluorescence intensity and the viral titers were stable at different passagedrecombinant PRRSV (Figure 3). Using the rSD16/TRS2/DsRed as a tool, the virus tracking in MARC-145 was observed by livecell imaging (Supplementary Movie 1). Quantification of the DsRed fluorescence positive cells was carried out by using flow cytometry, which shows it is a quick and reliable way to detect the level of PRRSV infection (Figure 4).

In conclusion, a recombinant HP-PRRSV carrying the DsRed gene as a separate transcription unit based on the BAC system was successfully rescued. The recombinant viruses can be useful for studying PRRSV transcription and replication by visualization. In addition, the strategy using a TRS2-derived foreign gene expression provides a new method for expressing foreign genes in the PRRSV genome. In the studies of PRRSV visualization, to ensure the fluorescent proteins not interfering with the natural 


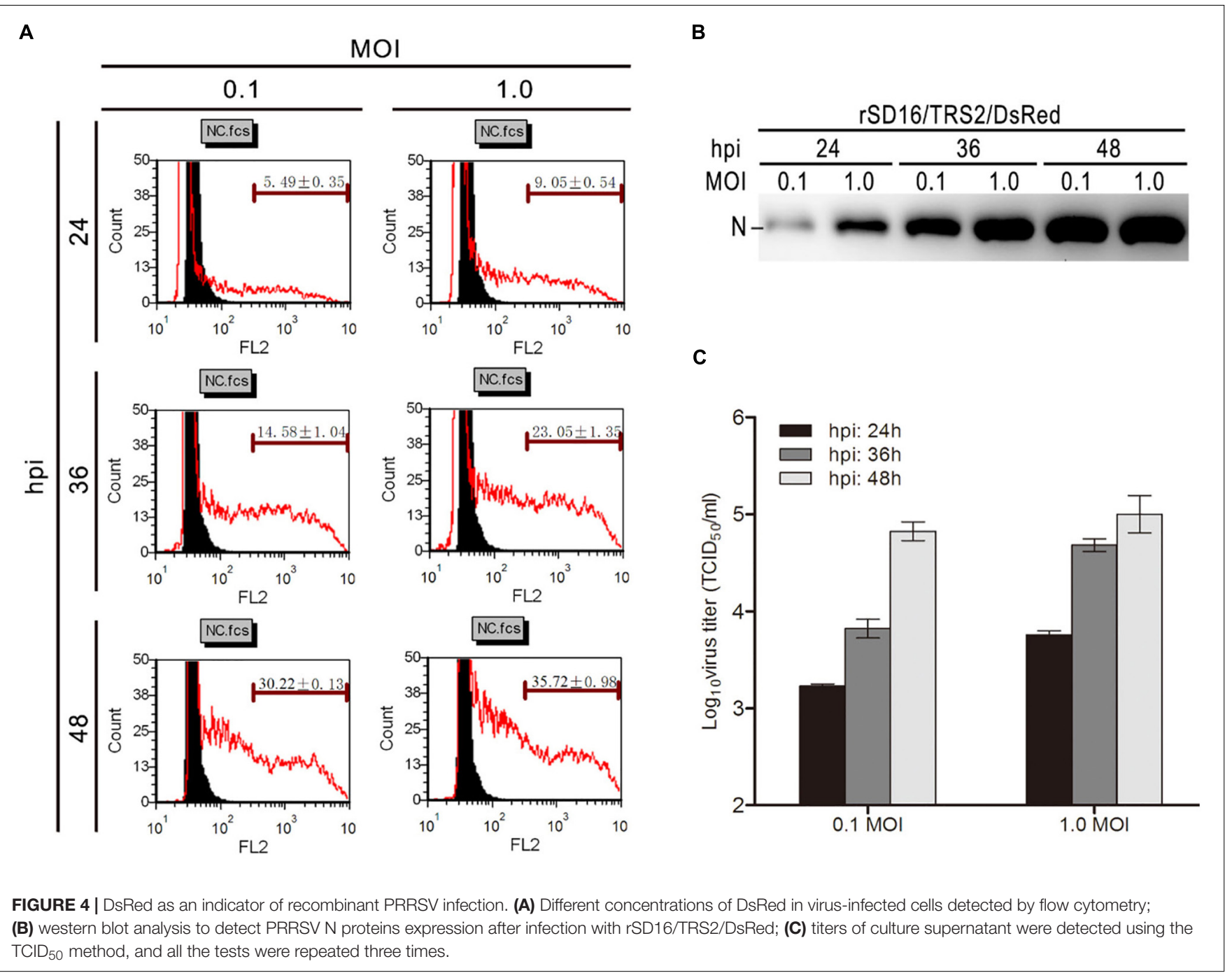

infection state of the virus is still the challenge, which was just as reported in the study of PRV (Yang et al., 2020). In the future, CRISPR technology to achieve viral nucleic acid labeling can explain the biological characteristics of the virus that are closest to the real states.

\section{DATA AVAILABILITY STATEMENT}

The original contributions presented in the study are included in the article/Supplementary Material, further inquiries can be directed to the corresponding authors.

\section{AUTHOR CONTRIBUTIONS}

$\mathrm{BH}, \mathrm{YaS}$, and $\mathrm{QZ}$ conceived and designed the experiments. $\mathrm{NL}, \mathrm{BH}$, and $\mathrm{YZ}$ performed the experiments. NL, LY, and YuS analyzed the data of experiments and drafted the manuscript. All authors contributed to the article and approved the submitted version.

\section{FUNDING}

This research was supported by the National Natural Science Foundation of China (Grant No. 31870917), the Program for Innovative Research Teams of Science and Technology in the University of Henan Province (Grant No. 20IRTSTHN024), and the Foundation of Nanyang Normal University (Grant No. 218264).

\section{SUPPLEMENTARY MATERIAL}

The Supplementary Material for this article can be found online at: https://www.frontiersin.org/articles/10.3389/fmicb.2022. 839845/full\#supplementary-material

Supplementary Movie 1 | Live imaging of rSD16/TRS2/DsRed observed by live-cell microscopy. MARC-145 cells were infected with rSD16/TRS2/DsRed at an $\mathrm{MOI}$ of 0.01 in a $35 \mathrm{~mm}$ cell culture dish. DsRed fluorescence signals were acquired with the time interval of $1 \mathrm{~min}$ for $3 \mathrm{~h}$ using a live-cell station, as described in Materials and methods. Images were processed into a movie of six frames $\mathrm{s}^{-1}$ by using QuickTime Pro. Scale bar, $50 \mu \mathrm{m}$. 


\section{REFERENCES}

Albina, E. (1997). Epidemiology of porcine reproductive and respiratory syndrome (PRRS): an overview. Vet. Microbiol. 55, 309-316. doi: 10.1016/s0378-1135(96) 01322-3

Almazan, F., Dediego, M. L., Galan, C., Escors, D., Alvarez, E., Ortego, J., et al. (2006). Construction of a severe acute respiratory syndrome coronavirus infectious cDNA clone and a replicon to study coronavirus RNA synthesis. J. Virol. 80, 10900-10906. doi: 10.1128/JVI.00385-06

Almazan, F., Gonzalez, J. M., Penzes, Z., Izeta, A., Calvo, E., Plana-Duran, J., et al. (2000). Engineering the largest RNA virus genome as an infectious bacterial artificial chromosome. Proc, Natl. Acad. Sci. U.S.A. 97, 5516-5521. doi: 10.1073/ pnas.97.10.5516

Amarilla, A. A., Sng, J. D. J., Parry, R., Deerain, J. M., Potter, J. R., Setoh, Y. X., et al. (2021). A versatile reverse genetics platform for SARS-CoV-2 and other positive-strand RNA viruses. Nat. Commun. 12:3431. doi: 10.1038/s41467-02123779-5

Behrens, R. T., Aligeti, M., Pocock, G. M., Higgins, C. A., and Sherer, N. M. (2017). Nuclear export signal masking regulates HIV-1 rev trafficking and viral RNA nuclear export. J. Virol. 91:e02107-16. doi: 10.1128/JVI.02107-16

Bernstein, H. D., Sarnow, P., and Baltimore, D. (1986). Genetic complementation among poliovirus mutants derived from an infectious cDNA clone. J. Virol. 60, 1040-1049. doi: 10.1128/JVI.60.3.1040-1049.1986

Borst, E. M., Hahn, G., Koszinowski, U. H., and Messerle, M. (1999). Cloning of the human cytomegalovirus (HCMV) genome as an infectious bacterial artificial chromosome in Escherichia coli: a new approach for construction of HCMV mutants. J. Virol. 73, 8320-8329. doi: 10.1128/JVI.73.10.8320-8329.1999

Charerntantanakul, W. (2012). Porcine reproductive and respiratory syndrome virus vaccines: immunogenicity, efficacy and safety aspects. World J, Virol. 1, 23-30. doi: 10.5501/wjv.v1.i1.23

Chaudhari, J., and Vu, H. L. X. (2020). porcine reproductive and respiratory syndrome virus reverse genetics and the major applications. Viruses 12:1245. doi: $10.3390 / \mathrm{v} 12111245$

Chiem, K., Morales Vasquez, D., Park, J. G., Platt, R. N., Anderson, T., Walter, M. R., et al. (2021). Generation and characterization of recombinant SARSCoV-2 expressing reporter genes. J. Virol. 95:e02209-20. doi: 10.1128/JVI. 02209-20

Davey, G. M., Mueller, S. N., van Vliet, C., Gigowski, M., Zaid, A., Davies, B., et al. (2013). Identification of a MHC I-restricted epitope of DsRed in C57BL/6 mice. Mol, Immunol. 53, 450-452. doi: 10.1016/j.molimm.2012.10.003

Fang, Y., Rowland, R. R., Roof, M., Lunney, J. K., Christopher-Hennings, J., and Nelson, E. A. (2006). A full-length cDNA infectious clone of North American type 1 porcine reproductive and respiratory syndrome virus: expression of green fluorescent protein in the Nsp2 region. J. Virol. 80, 11447-11455. doi: 10.1128/JVI.01032-06

Fang, Y., and Snijder, E. J. (2010). The PRRSV replicase: exploring the multifunctionality of an intriguing set of nonstructural proteins. Virus Res. 154, 61-76. doi: 10.1016/j.virusres.2010.07.030

Garcia-Parajo, M. F., Koopman, M., van Dijk, E. M., Subramaniam, V., and van Hulst, N. F. (2001). The nature of fluorescence emission in the red fluorescent protein DsRed, revealed by single-molecule detection. Proc. Nat. Acad. Sci. U.S.A. 98, 14392-14397. doi: 10.1073/pnas.251525598

Gonzalez, J. M., Penzes, Z., Almazan, F., Calvo, E., and Enjuanes, L. (2002). Stabilization of a full-length infectious cDNA clone of transmissible gastroenteritis coronavirus by insertion of an intron. J. Virol. 76, 4655-4661. doi: 10.1128/jvi.76.9.4655-4661.2002

Hawley, T. S., Telford, W. G., Ramezani, A., and Hawley, R. G. (2001). Four-color flow cytometric detection of retrovirally expressed red, yellow, green, and cyan fluorescent proteins. BioTechniques 30, 1028-1034. doi: 10.2144/01305rr01

Hendriks, G., Atallah, M., Raamsman, M., Morolli, B., van der Putten, H., Jaadar, H., et al. (2011). Sensitive DsRed fluorescence-based reporter cell systems for genotoxicity and oxidative stress assessment. Mutat. Res. 709-710, 49-59. doi: 10.1016/j.mrfmmm.2011.02.013

Hoenen, T., Groseth, A., de Kok-Mercado, F., Kuhn, J. H., and Wahl-Jensen, V. (2011). Minigenomes, transcription and replication competent virus-like particles and beyond: reverse genetics systems for filoviruses and other negative stranded hemorrhagic fever viruses. Antivir. Res. 91, 195-208. doi: 10.1016/j. antiviral.2011.06.003
Huang, Y. W., Fang, Y., and Meng, X. J. (2009). Identification and characterization of a porcine monocytic cell line supporting porcine reproductive and respiratory syndrome virus (PRRSV) replication and progeny virion production by using an improved DNA-launched PRRSV reverse genetics system. Virus Res. 145, 1-8. doi: 10.1016/j.virusres.2009.05.013

Ishibashi, K., Matsumoto-Yokoyama, E., and Ishikawa, M. (2017). A tomato spotted wilt virus S RNA-based replicon system in yeast. Sci. Rep. 7:12647. doi: 10.1038/s41598-017-12687-8

Jacobsen, H. K., Sleire, L., Wang, J., Netland, I. A., Mutlu, E., Forde, H., et al. (2013). Establishment of a novel dsRed NOD/Scid mouse strain to investigate the host and tumor cell compartments. Cancer Invest. 31, 221-230. doi: 10.3109/ 07357907.2013.780075

Kanai, Y., Kawagishi, T., Nouda, R., Onishi, M., Pannacha, P., Nurdin, J. A., et al. (2019). Development of stable rotavirus reporter expression systems. J. Virol. 93:e01774-18. doi: 10.1128/JVI.01774-18

Kanda, T., Yajima, M., Ahsan, N., Tanaka, M., and Takada, K. (2004). Production of high-titer Epstein-Barr virus recombinants derived from Akata cells by using a bacterial artificial chromosome system. J. Virol. 78, 7004-7015. doi: 10.1128/ JVI.78.13.7004-7015.2004

Li, Q., Wang, X., Wang, C., Yu, Y., Wang, G., Gao, J., et al. (2016). Intracellular expression of an anti-idiotypic antibody single-chain variable fragment reduces porcine reproductive and respiratory syndrome virus infection in MARC-145 cells. Antivir. Ther. 21, 161-170. doi: 10.3851/IMP2980

Li, Y., Wang, S., Zhu, H., and Zheng, C. (2011). Cloning of the herpes simplex virus type 1 genome as a novel luciferase-tagged infectious bacterial artificial chromosome. Arch. Virol. 156, 2267-2272. doi: 10.1007/s00705-011-1094-9

Messerle, M., Crnkovic, I., Hammerschmidt, W., Ziegler, H., and Koszinowski, U. H. (1997). Cloning and mutagenesis of a herpesvirus genome as an infectious bacterial artificial chromosome. Proc. Natl. Acad, Sci, U.S.A. 94, 14759-14763. doi: 10.1073/pnas.94.26.14759

Nielsen, H. S., Liu, G., Nielsen, J., Oleksiewicz, M. B., Botner, A., Storgaard, T., et al. (2003). Generation of an infectious clone of VR-2332, a highly virulent North American-type isolate of porcine reproductive and respiratory syndrome virus. J. Virol. 77, 3702-3711. doi: 10.1128/jvi.77.6.3702-3711.2003

Nouda, R., Minami, S., Kanai, Y., Kawagishi, T., Nurdin, J. A., Yamasaki, M., et al. (2021). Development of an entirely plasmid-based reverse genetics system for 12-segmented double-stranded RNA viruses. Proc. Natl. Acad. Sci. U.S.A. 118:e2105334118. doi: 10.1073/pnas.2105334118

Park, G. S., Lim, S. I., Hong, S. H., and Song, J. Y. (2012). Establishment and characterization of an infectious cDNA clone of a classical swine fever virus LOM strain. J. Vet. Sci. 13, 81-91. doi: 10.4142/jvs.2012.13.1.81

Racaniello, V. R., and Baltimore, D. (1981). Cloned poliovirus complementary DNA is infectious in mammalian cells. Science 214, 916-919. doi: 10.1126/ science.6272391

Sang, Y., Shi, J., Sang, W., Rowland, R. R., and Blecha, F. (2012). Replicationcompetent recombinant porcine reproductive and respiratory syndrome (PRRS) viruses expressing indicator proteins and antiviral cytokines. Viruses 4, 102-116. doi: 10.3390/v4010102

Shizuya, H., Birren, B., Kim, U. J., Mancino, V., Slepak, T., Tachiiri, Y., et al. (1992). Cloning and stable maintenance of 300-kilobase-pair fragments of human DNA in Escherichia coli using an F-factor-based vector. Proc. Natl. Acad. Sci. U.S.A. 89, 8794-8797. doi: 10.1073/pnas.89.18.8794

St-Jean, J. R., Desforges, M., Almazan, F., Jacomy, H., Enjuanes, L., and Talbot, P. J. (2006). Recovery of a neurovirulent human coronavirus OC43 from an infectious cDNA clone. J. Virol. 80, 3670-3674. doi: 10.1128/JVI.80.7.36703674.2006

Strickfaden, H., Zunhammer, A., van Koningsbruggen, S., Kohler, D., and Cremer, T. (2010). 4D chromatin dynamics in cycling cells: theodor Boveri's hypotheses revisited. Nucleus 1, 284-297. doi: 10.4161/nucl.1.3.11969

Suter, M., Lew, A. M., Grob, P., Adema, G. J., Ackermann, M., Shortman, K., et al. (1999). BAC-VAC, a novel generation of (DNA) vaccines: a bacterial artificial chromosome (BAC) containing a replication-competent, packaging-defective virus genome induces protective immunity against herpes simplex virus 1. Proc. Natl. Acad. Sci, U S.A. 96, 12697-12702. doi: 10.1073/pnas.96.22.12697

Tian, K., Yu, X., Zhao, T., Feng, Y., Cao, Z., Wang, C., et al. (2007). Emergence of fatal PRRSV variants: unparalleled outbreaks of atypical PRRS in China and molecular dissection of the unique hallmark. PLoS One 2:e526. doi: 10.1371/ journal.pone. 0000526 
Truong, H. M., Lu, Z., Kutish, G. F., Galeota, J., Osorio, F. A., and Pattnaik, A. K. (2004). A highly pathogenic porcine reproductive and respiratory syndrome virus generated from an infectious cDNA clone retains the in vivo virulence and transmissibility properties of the parental virus. Virology 325, 308-319. doi: 10.1016/j.virol.2004.04.046

Wang, C., Huang, B., Kong, N., Li, Q., Ma, Y., Li, Z., et al. (2013). A novel porcine reproductive and respiratory syndrome virus vector system that stably expresses enhanced green fluorescent protein as a separate transcription unit. Vet. Res. 44:104. doi: 10.1186/1297-9716-44-104

Wang, X., Cato, P., Lin, H. C., Li, T., Wan, D., Alcocer, M. J., et al. (2014). Optimisation and use of humanised RBL NF-AT-GFP and NF-AT-DsRed reporter cell lines suitable for high-throughput scale detection of allergic sensitisation in array format and identification of the ECM-integrin interaction as critical factor. Mol. Biotechnol. 56, 136-146. doi: 10.1007/s12033-013-9689-x

Wensvoort, G., Terpstra, C., Pol, J. M., ter Laak, E. A., Bloemraad, M., de Kluyver, E. P., et al. (1991). Mystery swine disease in The Netherlands: the isolation of Lelystad virus. Vet. Q. 13, 121-130. doi: 10.1080/01652176.1991.9694296

Wolff, H., Hadian, K., Ziegler, M., Weierich, C., Kramer-Hammerle, S., Kleinschmidt, A., et al. (2006). Analysis of the influence of subcellular localization of the HIV Rev protein on Rev-dependent gene expression by multi-fluorescence live-cell imaging. Exp. Cell Res. 312, 443-456. doi: 10.1016/ j.yexcr.2005.11.020

Yang, Y. B., Tang, Y. D., Hu, Y., Yu, F., Xiong, J. Y., Sun, M. X., et al. (2020). Single virus tracking with quantum dots packaged into enveloped viruses using CRISPR. Nano Lett. 20, 1417-1427.

Yu, Z., Zhou, J., and Hoffman, R. M. (2012). Lentivirus-based DsRed-2-transfected pancreatic cancer cells for deep in vivo imaging of metastatic disease. Methods Mol. Biol. 872, 69-83.
Zhang, S., Zhou, Y., Jiang, Y., Li, G., Yan, L., Yu, H., et al. (2011). Generation of an infectious clone of HuN4-F112, an attenuated live vaccine strain of porcine reproductive and respiratory syndrome virus. Virol. J. 8:410. doi: 10.1186/1743422X-8-410

Zhang, X., Li, C., and Gong, Z. (2014). Development of a convenient in vivo hepatotoxin assay using a transgenic zebrafish line with liverspecific DsRed expression. PLoS One 9:e91874. doi: 10.1371/journal.pone.009 1874

Conflict of Interest: YuS was employed by Shijiazhuang Customs (Huanghua Port).

The remaining authors declare that the research was conducted in the absence of any commercial or financial relationships that could be construed as a potential conflict of interest.

Publisher's Note: All claims expressed in this article are solely those of the authors and do not necessarily represent those of their affiliated organizations, or those of the publisher, the editors and the reviewers. Any product that may be evaluated in this article, or claim that may be made by its manufacturer, is not guaranteed or endorsed by the publisher.

Copyright (c) 2022 Li, Zhang, Yao, Shi, Zhao, Huang and Sun. This is an open-access article distributed under the terms of the Creative Commons Attribution License (CC BY). The use, distribution or reproduction in other forums is permitted, provided the original author(s) and the copyright owner(s) are credited and that the original publication in this journal is cited, in accordance with accepted academic practice. No use, distribution or reproduction is permitted which does not comply with these terms. 